\title{
Interaction of short and intense laser pulses with dielectric materials: from absorption to ablation
}

\author{
Guillaume Duchateau, Benoît Chimier, and Vladimir Tikhonchuk \\ Université de Bordeaux-CNRS-CEA, Centre Lasers Intenses et Applications, UMR 5107, 351 Cours de la Libération, 33405 Talence, France \\ duchateau@celia.u-bordeaux1.fr ; chimier@celia.u-bordeaux1.fr ; tikhonchuk@celia.u-bordeaux1.fr
}

\begin{abstract}
Modeling of the interaction of short laser pulses with dielectric materials is addressed. Based on phenomenological considerations, it is shown how the transition from primary absorption to ablation takes place. This description is illustrated by two particular examples allowing to highlight specific physical phenomena. An accurate estimate of the early absorption, which strongly depends on material characteristics, is shown to play an important role. An approach to evaluate this early absorption is presented.
\end{abstract}

With the development of short and intense laser pulses, laser modifications of materials for various applications become possible. Such modifications are due to energy absorption which ultimately leads to ablation for the highest laser intensities. The design of specific nano-structures requires both the knowledge and the modelling of physical mechanisms responsible for these material modifications. In this presentation, we focus on dielectric materials. In that case, briefly, the physical processes which may lead to material modifications are the following: ionization (electron transition from the valence band to the conduction band), heating of conduction electrons, energy transfer from electrons to the lattice, material response relying mainly on hydrodynamics [1]. Regarding the electron dynamics, it can be described in terms of collisions including the electron-photon, electron-ion-photon, electronphonon-photon, electron-ion, electron-phonon, and electron-electron collisions [2].

Based on this general phenomenology, we will consider two particular examples under simplified conditions in order to highlight specific mechanisms. The fist study is devoted to the evolution of surface damage and ablation of silica with respect to the pulse duration within the sub-picosecond timescale. This study exhibits the role of various ionization mechanisms depending on the pulse duration. The second study is devoted to the interaction of subpicosecond laser pulses with dielectric nanoparticles embedded in optical materials. It exhibits the influence of possible laser field enhancement leading to a strong nonlinear increase in the density of conducting electrons even without impact ionization.
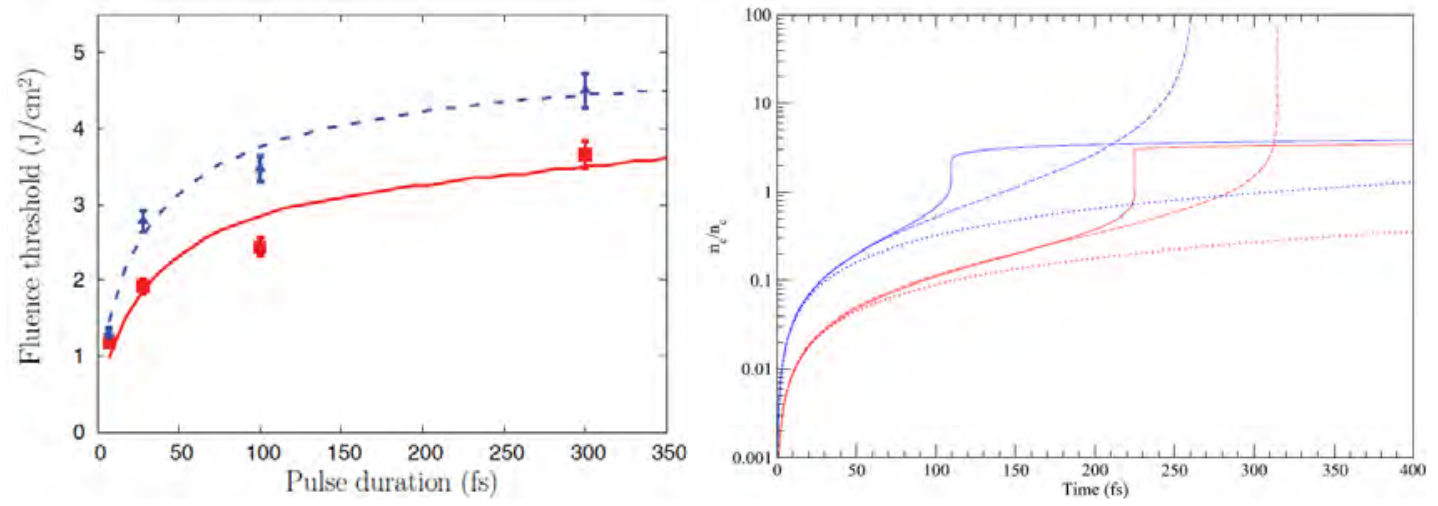

Fig. 1. (Left) Evolution of the fluence threshold for damage (red) and ablation (blue) as experimentally observed (square and triangle) and theoretically predicted (solid line) with respect to the pulse duration [3]. (Right) Evolution of the electron density in the conduction band as a function of time in dielectric nanoparticles where a laser field enhancement takes place [4].

1) To provide valuable information on the dielectric material response to surface laser irradiation within a broad range of pulse durations (from a few-cycle to sub-picosecond pulses), the damage and ablation thresholds were measured. A model incorporating the absorption of the laser energy in the material, and its subsequent relaxation, was developed including relevant criteria of damage and ablation thresholds, which were determined consistently with experimental observations of the laser irradiated zone [3]. Numerically, the damage threshold is related to the lattice melting temperature, and the ablation is induced when the free electron energy overcomes the ion binding energy (electron-driven ablation process). 
The damage and ablation thresholds at the surface of fused silica are estimated and compared to experimental results for a $800 \mathrm{~nm}$ laser with pulse durations ranging from 7 to $300 \mathrm{fs}$ (see Fig. 1 (left)). The importance of various ionization processes is investigated, and results show that the impact ionization plays a role whatever the interaction regime is. Its relative importance in material ionization gradually increases with pulse duration. It is also shown that both damage and ablation thresholds tend to coincide in the ultrashort regime ( $7 \mathrm{fs}$ ) due to the sharp increase of the free electron density, which strongly reduces the cohesion limit of the material under strong non-equilibrium transient conditions.

2) The interaction of a dielectric nanoparticle, embedded in a bulk of an optical dielectric material, with an intense and short laser pulse will be also presented [4]. Due to the finite size of the target and the possible large production of electrons in the conduction band, a large electric field enhancement or intensity overshoot may be induced inside the particle. Since ionization rates also depend on the instantaneous electric field, a strong timedependent connection between free electron production and intensity enhancement may take place. Such a connection is shown to lead to a nonlinear temporal increase in the free electron density (see Fig. 1 (right)), called optical avalanche, similar to the one induced by electron impact ionization. However, the present build-up in the electron density clearly exhibits more nonlinear features than standard collisional avalanche, which is shown to induce an exponential growth of the density: when the optical avalanche is engaged, the temporal electron evolution exhibits an explosive behavior.

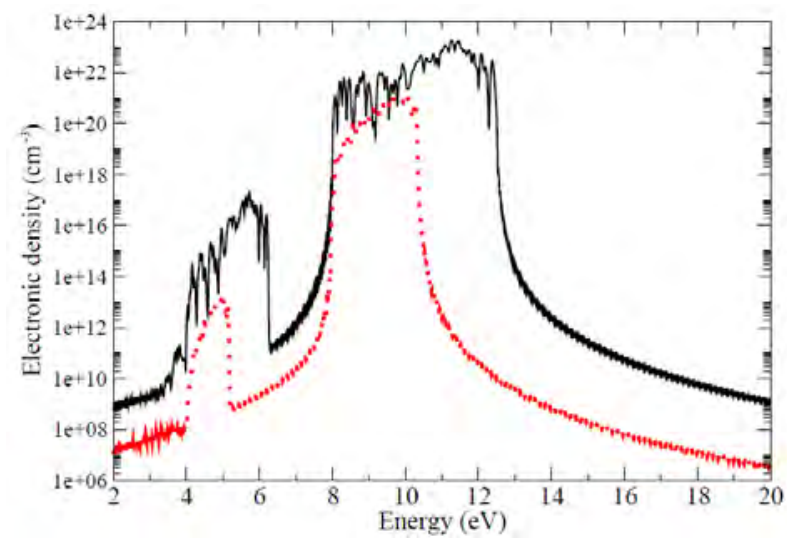

Fig. 2. Laser-induced electron density in the conduction band of $\mathrm{NaCl}$ (black curve) and KDP (red curve) crystals as a function of the photon energy [5]. Both different behaviors show the influence of real material characteristics.

Both these examples highlight that the simplified modeling approaches are limited due to unknown physical parameters driving the electron dynamics. In particular, depending on the studied material, an accurate evaluation of the multiphoton absorption cross section, which initiates the laser-matter interaction, is important. To do so, we will present a semi-analytical model for free electron production induced by multiphoton ionization in dielectric materials [5]. Within this approach, the laser induced absorption is described through the Bloch-Volkov formalism, and the electronic structure of materials is evaluated through first-principle calculations. Results obtained for $\mathrm{NaCl}$ and $\mathrm{KDP}\left(\mathrm{KH}_{2} \mathrm{PO}_{4}\right)$ materials show that significant deviations from the parabolic band approximation may occur (see Fig. 2). When the laser intensity increases, high multiphotonic orders may become the predominant mechanisms out of the centre of the Brillouin Zone. Also, this study clearly shows the importance of taking into account the whole physical characteristics of the material of interest including in particular its real band structure.

[1] S. Juodkazis, K. Nishimura, S. Tanaka, H. Misawa, E. G. Gamaly, B. Luther-Davies, L. Hallo, P. Nicolai, and V.T. Tikhonchuk, "LaserInduced Microexplosion Confined in the Bulk of a Sapphire Crystal: Evidence of Multimegabar Pressures,” Phys. Rev. Lett. 96, 166101 (2006).

[2] A. Kaiser, B. Rethfeld, M. Vicanek, and G. Simon, "Microscopic processes in dielectrics under irradiation by subpicosecond laser pulses," Phys. Rev. B 61, 11437 (2000).

[3] B. Chimier et al, “Damage and ablation thresholds of fused-silica in femtosecond regime,” Phys. Rev. B 84, 094104 (2011).

[4] G. Duchateau, "Strong nonlinear electron multiplication without impact ionization in dielectric nanoparticles embedded in optical materials," Phys. Plasmas 20, 022306 (2013).

[5] C. Mézel, G. Duchateau, G. Geneste, and B. Siberchicot, “A model for multiphoton absorption in dielectric materials induced by intense and short laser pulses,” submitted to J. Phys: condens. Matter. 